\title{
Computer-Based Tutorial to Enhance the Informed Consent Process for Cataract Surgery in Serbian- or Turkish-Speaking Patients
}

\author{
Manuel Ruiss ${ }^{a}$ Oliver Findl $^{a} \quad$ Ana Prinz $^{a} \quad$ Guenal Kahraman $^{b}$ Sava Barisic ${ }^{c}$ \\ Orkun Muftuoglud Nino Hirnschalla \\ aDepartment of Ophthalmology, Vienna Institute for Research in Ocular Surgery, Hanusch Hospital, Vienna, Austria; \\ bepartment of Ophthalmology, Academic Teaching Hospital of Saint John of God, Vienna, Austria; 'Eye Clinic, \\ Clinical Center of Vojvodina, Novi Sad, Serbia; ${ }^{\mathrm{d} D e p a r t m e n t}$ of Ophthalmology, Koc University, School of Medicine, \\ Istanbul, Turkey
}

\section{Keywords}

Informed consent process · Cataract surgery · Catlnfo tool · Serbian · Turkish

\begin{abstract}
Introduction: This study analyses if a translated version of the Catlnfo tool increases the knowledge of Serbian- and Turkish-speaking patients about cataract surgery. Methods: In total, 61 cataract patients, literate in Serbian or Turkish, were randomly allocated into two groups. Via an interactive computer-based tool ("Catlnfo"), patients either saw a detailed audio-visual presentation about cataract surgery (study group) or a "placebo" video (control group). Afterwards, all participants had a face-to-face discussion with an ophthalmologist. Immediately after the interview and on the day of surgery, all patients had to fill out a questionnaire including knowledge and demographic questions. Patients in the study group were further asked about their satisfaction with the CatInfo tool and the usefulness of such a device before other interventions. Results: Patients in the study group answered significantly more questions correctly compared to the control group, and this information gain remained stable over a 1-week period. There was a significant low nega-
\end{abstract}

karger@karger.com www.karger.com/ore

Karger"

BOPEN ACCESS
(C) 2021 The Author(s)

Published by S. Karger AG, Basel

This is an Open Access article licensed under the Creative Commons Attribution-NonCommercial-4.0 International License (CC BY-NC) (http://www.karger.com/Services/OpenAccessLicense), applicable to the online version of the article only. Usage and distribution for commercial purposes requires written permission. tive correlation between educational level and the test results, whereas age and computer habits of the participants did not have an influence on correct answers. Satisfaction with the CatInfo tool was high in the Serbian and the Turkish group ( $96 \%$ and $84 \%$, respectively), and $92 \%$ of Serbian patients as well as $62 \%$ of the Turkish patients rated that they could imagine to use such a device before any other surgery. Discussion/Conclusion: The translated version of the Catlnfo tool improved patients' knowledge, and this information gain remained stable until the day of the surgery.

\footnotetext{
(c) 2021 The Author(s)

Published by S. Karger AG, Basel
}

\section{Introduction}

Informing a patient about an invasive procedure and obtaining informed consent is a legal and ethical requirement [1]. It is necessary for valid consent that the patient has agreed to the surgery voluntarily after a sufficient consideration time, and this includes that he is conscious about and understood the purpose, procedure, alternatives, benefits, and risks of the medical treatment properly [1-3]. However, it was shown that patients are often 
inadequately informed and that their comprehension of the surgical procedure is poor $[4,5]$. Reasons for that, among others, could be the increasing complexity of the medical interventions, growing lack of time of the medical staff, higher demands of patients for more extensive information, and language barriers $[3,6]$. One reason to overcome the latter problem would be the involvement of professional medical interpreters in the patient-physician interview. However, it was shown that time constraints and lack of immediate availability of interpreters are the main reasons for physicians not using these services $[3,7]$.

One possible solution would be a multimedia tool for informing patients about the planned surgery in their mother tongue. In a previous study, we have developed and evaluated a computer-based tutorial about cataract surgery, the so-called "CatInfo tool." It was shown that patients who used this computer-based tutorial additionally to a face-to-face interview with an ophthalmologist were significantly better informed than patients seeing a placebo presentation and only being informed by a physician [8]. The overall satisfaction with the device was high. Meanwhile, the German version of the CatInfo tool is part of our daily routine additionally to the face-to-face interview with an ophthalmologist.

A study performed in gynaecological departments in Switzerland showed that one of the main reasons of discontent with the informed consent process for surgical interventions was Turkish or Serbo-Croat language [9]. According to the 2019 yearly migration report of the Federal Ministry for European and International Affairs of the Republic of Austria, Serbian and Turkish citizens are the largest group of non-native German-speaking people living in Austria [10]. Therefore, the CatInfo tool was translated into Serbian and Turkish and validated in this clinical trial.

\section{Materials and Methods}

For this randomized controlled patient- and examiner-masked single-centre study, patients older than 21 years with cataract and who were literate in Serbian (Serbian group) or Turkish (Turkish group) were recruited in the eye department of the Hanusch hospital, Vienna. Exclusion criteria were visual acuity of $<6 / 60$ Snellen in the worse eye, severe hearing loss, previous ophthalmic surgery, and inability to use a touchscreen device. Furthermore, participants were excluded if they were not literate in German (both groups), since a proper understanding in German was necessary to understand and to be able to sign the informed consent for the study. All the research and measurements complied with the tenets of the Declaration of Helsinki and were approved by the ethics committee of the city of Vienna (Approval Reference No. EK16-258-VK). Written informed consent was obtained from all patients before enrolment in the study and before any study measures were performed. The study was registered at clinicaltrials.gov (https://clinicaltrials.gov) with the clinical trial registration number NCT03748368.

\section{CatInfo Tool}

The CatInfo tool [8] is a software covering the topics cataract, surgery, risks, and complications and is presented in an audio-visual fashion on a touchscreen device with headphones. The patient is guided through the program in a stepwise approach (small chapters) by animated two-dimensional and some three-dimensional illustrations. The patient's feedback is required after each chapter. A "traffic light" system is used, where green means "continue, everything understood," yellow means "I have further questions, that I would like to discuss with a physician afterwards," and red means "I would like to repeat the last module again." All the patient's feedback for each chapter was recorded in a log-documentation. However, no printout of the log-documentation was used in this study because of the masking of the ophthalmologist performing the face-to-face discussion.

\section{Knowledge Questionnaire}

The questionnaire was already used in a previous study [8] and contained 23 multiple choice questions about the patient's surgery-related knowledge, for example, symptoms, surgical procedure, risks, complications, intraocular lens design, and postoperative period. These questions were developed by a group of ophthalmologists and patients that had never seen the CatInfo tool before.

Furthermore, there were also questions about demographic and social background and (in the study group only) a visual analogue scale (VAS) for evaluating the CatInfo tool. Patients had to rate how satisfied they were with the device and if they would find it useful to use such a learning device before any other surgery on a scale between 0 and $10(0=$ absolutely not and $10=$ absolutely $)$. Of the 23 multiple choice questions, 15 had 4 response options, of which only 1 was correct ( 1 point for each correct answer), whereas the other 8 questions had 2 response options (yes/no; 0.5 points for each correct answer). Hence, the maximal score was 19 points. No points were given for incorrect or missing answers.

\section{Translation Procedure}

The CatInfo tool and the knowledge questionnaire were translated into Serbian and Turkish by native speakers, who are certified ophthalmologists and bilingual. In a second step, the Serbian and Turkish versions were backtranslated by two different certified bilingual ophthalmologists to detect any potential translation problems. The translated version of the CatInfo tool was then presented to a Serbian and a Turkish focus group. The focus group consisted of 5 patients per language, who recently underwent cataract surgery. Feedback of the focus group was then implemented.

\section{Evaluation Study}

After obtaining informed consent of a patient to participate in the study, group allocation into the study or placebo group was performed using an online randomisation tool (https://www.randomizer.org). The CatInfo tool was only shown to the patients in the study group, whereas participants of the control group watched a sham presentation containing information about the Hanusch hospital and its history, but no cataract-relevant information. In all cases, this was followed by a standardized face-to-face interview 
Table 1. Comparison of the questionnaire score between the two groups and the two study visits

\begin{tabular}{lccl}
\hline & Control group & Study group & $\begin{array}{l}p \text { value } \\
\text { (between groups) }\end{array}$ \\
\hline Screening & $14(6-18)$ & $15.5(11-19)$ & 0.007 \\
Preoperative & $13.5(3-18.5)$ & $16.3(7.5-19)$ & 0.001 \\
$p$ value (between visits) & 0.638 & 0.847 & \\
\hline
\end{tabular}

Statistical significance between the groups was tested using the Mann-Whitney U test and between the visits with the Wilcoxon signed-rank test. $p<0.05$ was considered significant.

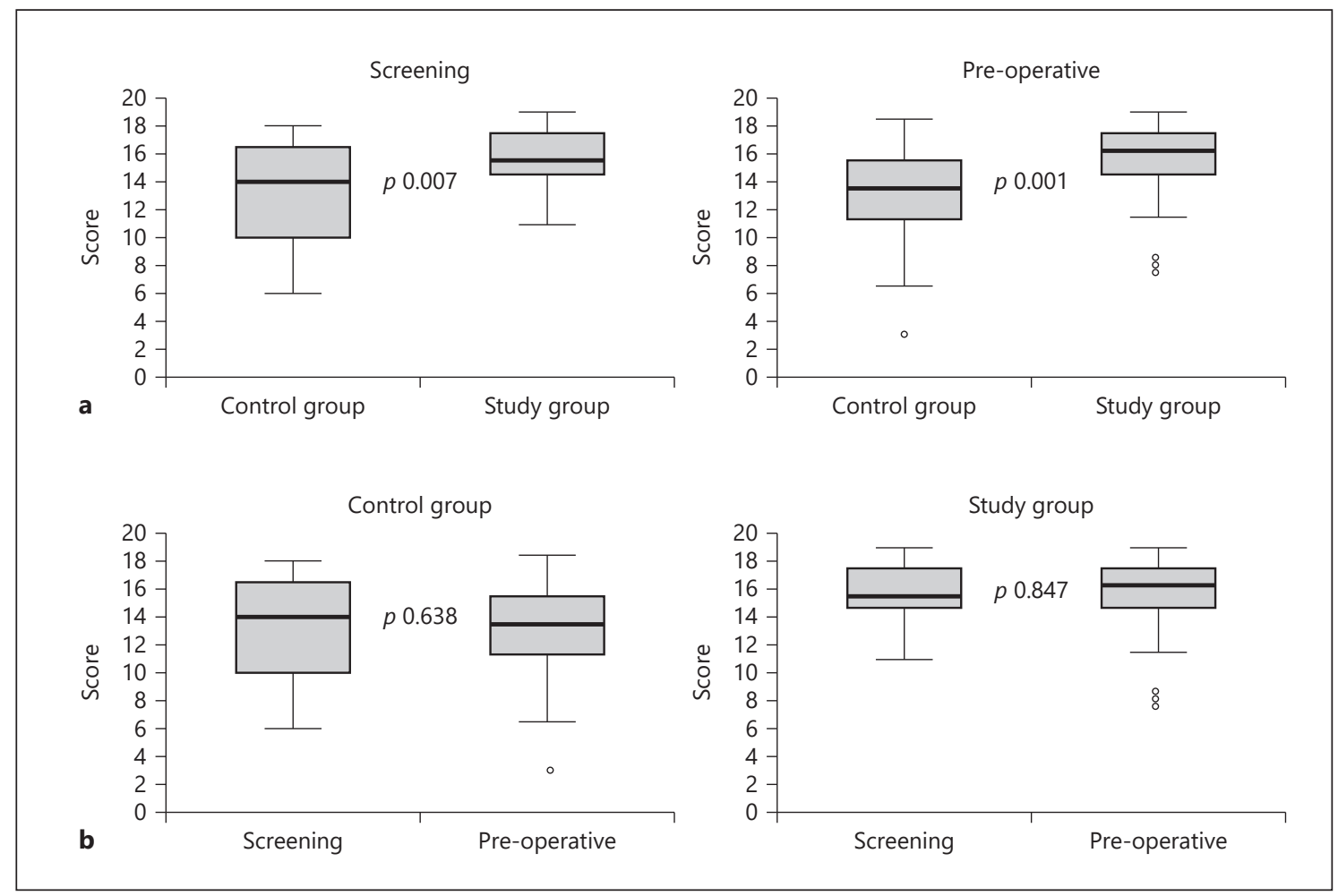

Fig. 1. Correct answers in the questionnaire between study groups (a) and study visits (b).

with an ophthalmologist, who was masked to group allocation. Afterwards, both groups received the knowledge questionnaire in either Serbian or Turkish. This questionnaire was given to the patients a second time on the day of surgery.

\section{Statistical Analysis}

All statistical analyses were performed using Excel 2016 (Microsoft Corporation, USA) and SPSS Statistics Version 21 for Mac (IBM, USA). Descriptive data are shown as median and range. A $p$ value $<0.05$ was considered statistically significant.

To compare differences in questionnaire scores between the control and study group, the Mann-Whitney U test was applied and Wilcoxon signed-rank test to compare correct answers between both study visits. The association between age and test scores was analysed using the Spearman rank correlation coeffi- cient. Comparison between educational level as well as computer usage between both groups and correlation analysis between these factors and correctly answered questions was analysed with Kendall-Tau-b coefficient $(\tau)$. Fisher exact test was used to analyse if patients who are owners of a personal computer (PC) had higher VAS scores than patients who do not own a PC.

\section{Results}

Seventy patients were included in this study of which 9 patients had to be excluded: 6 patients refused to fill out the first or the second questionnaire, and the surgery of 3 


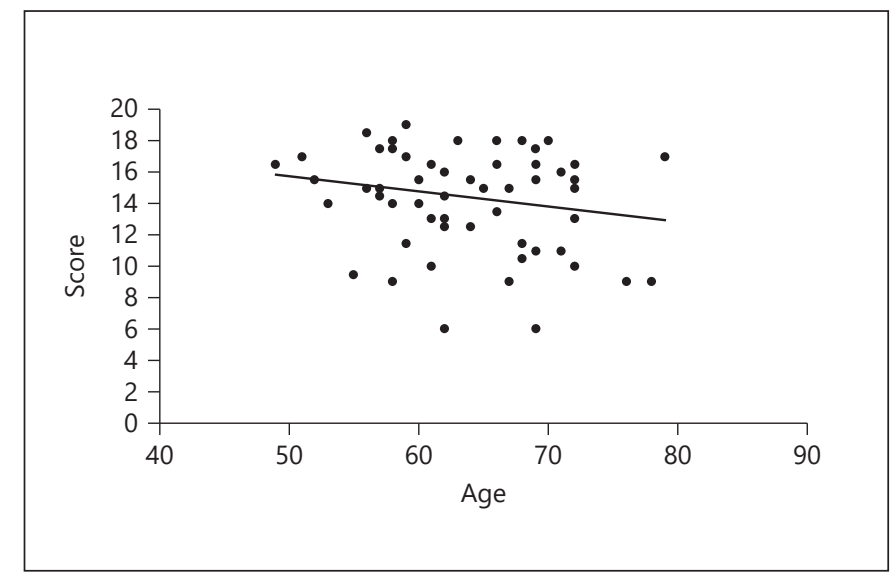

Fig. 2. Correlation between questionnaire score and age. Only the scatterplot for the screening visit is shown.

patients was cancelled. Hence, a total of 61 participants (36 in the Serbian group and 25 in the Turkish group) were included in this study. In total, $35 \%$ of the patients were female and $65 \%$ were male. The median age of the Serbian group was 65 years (range: $49-78$ years) and 62 years (range: 53-72 years) in the Turkish group.

At the screening visit, patients in the study group yielded a median score of 15.5 out of 19 points (range: $11-19$ points) compared to 14 points (range: $6-18$ points) in the control group ( $p$ 0.007). Similar results were found at the day of the surgery. However, no significant differences were found in the scores of the questionnaire between screening and preoperative visit in both groups (shown in Table 1; Fig. 1).

There was a small negative non-significant correlation between age of the participants and their test scores (screening visit: $r_{\mathrm{s}}-0.163, p 0.210$; preoperative visit: $r_{\mathrm{s}}$ $-0.217, p$ 0.093) (shown in Fig. 2). The same was found for educational level $(\tau-0.121, p 0.318)$ and computer usage $(\tau-0.124, p 0.286)$ between control and study groups (shown in Table 2). Furthermore, low correlation was found between highest graduation level (screening visit: $\tau 0.263, p 0.008$; preoperative visit: $\tau 0.199, p 0.046$ ) as well as computer habits (screening visit: $\tau 0.152, p 0.132$; preoperative visit: $\tau 0.053, p 0.597$ ) of patients and their questionnaire results, with the latter being non-significant.

Median satisfaction level with the CatInfo tool in the Serbian group was $96 \%$ (range: $66-100 \%$ ) and $84 \%$ (range: $50-100 \%)$ in the Turkish group. Higher satisfaction scores were achieved by owners of a PC compared to nonPC users ( $75 \%$ vs. $58 \%$ rated $100 \%$ satisfaction; Fisher's exact test: $8.842, p$ 0.026). Patients in the Serbian and
Table 2. Educational level and computer usage between control and study groups

\section{Control group, $\% \quad$ Study group, $\%$}

$\begin{array}{lrr}\text { Educational level } & & \\ \text { Secondary school } & 22 & 36 \\ \text { Apprenticeship } & 45 & 32 \\ \text { Vocational school } & 15 & 25 \\ \text { High school } & 6 & 7 \\ \text { University } & 12 & 0\end{array}$

$\begin{array}{lrr}\text { Computer use } & \\ \text { Rarely } & 30 & 54 \\ \text { Never } & 25 & 7 \\ \text { Weekly } & 15 & 7 \\ \text { Daily } & 30 & 32\end{array}$

Turkish group rated their readiness of using such a learning device before any other surgery with a median of $92 \%$ (range: $41-100 \%$ ) and 62\% (range: $0-100 \%$ ), respectively. Again, there was a significant difference between PC owners and non-PC users ( $81 \%$ vs. $55 \%$ rated $100 \%$ readiness; Fisher's exact test: 9.767, p 0.019).

\section{Discussion/Conclusion}

Patients who are Serbian/Turkish native speakers were better informed when using the CatInfo tool compared to those who only had a face-to-face informed consent discussion with a physician. In a previous study using the German version of the CatInfo tool, similar results were observed [8].

Improvement of the informed consent process before cataract surgery with the help of multimedia interventions has been shown in different studies [11-13] and has previously been reported with the German version of the CatInfo tool [8]. However, for patients with limited English proficiency, it was shown that a bedside interpreter phone system led to improvement of the informed consent process [7]. On the other hand, in non-native English-speaking patients undergoing cholecystectomy, addition of a translated PowerPoint presentation did not increase the understanding of the patient about the procedure [2]. Reason could be the difference in the setting, as Clark et al. [2] showed the patients the PowerPoint slides after the face-to-face interview.

Additionally, to prove that patients undergoing a CatInfo tool in the native language had improved knowledge, this study also showed that the knowledge gain in the 
study group did not change between screening visit and the second visit at the day of the surgery. Therefore, the translated version of the CatInfo tool was effective in improving the knowledge of the patients, and the information gain did not decrease during the 1-week period until the day of the surgery. Karan et al. [11] reported that the information gain, after using a multimedia information device for cataract surgery, remained stable directly and 1 day after the procedure. In our study, knowledge did not decrease in the time from screening until the day of the surgery, which is typically 1 week in our hospital. In patients undergoing knee arthroscopy, computer-based intervention-gained information was stable even 6 weeks after surgery [14], whereas with a multimedia information module for gynaecological pelvic pain laparoscopy, superior knowledge scores were not stable until 6 weeks after surgery [15]. Again, in the latter study, patients saw the multimedia intervention after the patient-clinician discussion. A review found that written or audio-visual materials for the informed consent process improved knowledge of procedures immediately (up to $24 \mathrm{~h}$ ), short term (1-14 days), and long term (>15 days) [1]. Hence, for further studies, it would be interesting to analyse knowledge increase over a longer time period and to use a cross-over design to assess if information gain depends on whether the interactive device is presented before or after the patient-ophthalmologist interview.

The outcomes of the questionnaire in our study did not depend on the age and computer habits, and only a small negative correlation was found for the educational levels of the patients. A previous study with the German version of the CatInfo tool showed similar results, and age and education of the patients showed only a low nonsignificant relationship with the test scores [8]. This was also confirmed in other studies, where age of participants did not have an influence on the questionnaire results in patients using a multimedia-based information program for cholecystectomy [16]. In another study, a tendency toward decreased knowledge was found with increasing age in patients watching a video presentation before cataract surgery [13]. Another study also found dependency of improved comprehension on age and education in patients using the iMedConsent platform [5].

Satisfaction with the translated version of the CatInfo tool was high in Serbian and Turkish patients, and participants in the Serbian group would also find it very helpful to use such an audio-visual information platform before any other surgery, whereas this was only moderate in the Turkish group. Furthermore, patients that owned a PC rated satisfaction and readiness to use the device be-

CatInfo Tool for Serbian- and Turkish-

Speaking Patients fore any other intervention higher than those who did not have access to a PC. This means that special attention should be drawn to patients who are not aware of using a multimedia device and could, therefore, need guidance in using a tablet. On the other hand, in the study with the German version of the CatInfo tool, correlation between computer literacy and patient satisfaction was low [8].

A good understanding of the surgical procedure to treat cataract is a prerequisite in the patient's decisionmaking in order to give informed consent. An additional audio-visual presentation, like the CatInfo tool, to the routine informed consent discussion might increase the rate of patients participating in the surgery. Further, in a preoperative situation, the patient usually is emotionally stressed and may have difficulties in the comprehension of the information that is being presented to him/her. Additional methods to improve the informed consent discussion could help the patient to repeat all necessary information at a speed suitable for the patient. Furthermore, the CatInfo tool offers the option to print the feedback of each patient, so that the physician is able to tailor the doctor-patient conversation to the patient's needs. Another advantage of an improved informed consent is that a patient is more likely to understand the severity of postoperative symptoms. This could lead to a reduced number of unnecessary visits in the emergency unit and more importantly to earlier visits in the case of relevant symptoms. All attempts to improve patient's knowledge about medical procedures could make patients feel more comfortable and thus being more adhered to the clinic. A next step will be to customize the CatInfo tool for patients with additional diseases and, hence, increased risk of postsurgical complications such as high myopia or pseudoexfoliation syndrome.

To summarize, the translated version of the CatInfo tool improved patient knowledge about cataract surgery, and this information remained stable until the day of the surgery. A translated interactive information platform additionally to the clinician-patient informed consent discussion may be a simple and effective way of better informing patients with language barriers, when an interpreter is not available. However, care should be taken if a patient is not experienced in using electronic devices.

\section{Acknowledgments}

The CatInfo software was designed and technical support was provided by Guido Schulte, Curt-Wilhelm Flakowski, and Rene Paradies. 


\section{Statement of Ethics}

All research and measurements followed the tenets of the Declaration of Helsinki and were approved by the Local Ethics Committee of the city of Vienna (Approval Reference No. EK16-258VK). Written informed consent was obtained from all patients before enrolment in the study and before any study measures were performed. The study was registered at clinicaltrials.gov (https:// clinicaltrials.gov) with the clinical trial registration number NCT03748368.

\section{Conflict of Interest Statement}

O. Findl is a scientific advisor to Alcon, Croma, Carl Zeiss Meditec AG, Johnson \& Johnson, and Merck, and N. Hirnschall has a research contract with Carl Zeiss Meditec AG and Hoya, but both do not have any financial interests related to the study. The authors have no proprietary or financial interest in any of the materials or equipment mentioned in this study.

\section{Author Contributions}

M. Ruiss conceptualised the study, performed data acquisition and statistical analysis, and prepared the manuscript; O. Findl conceptualised the study and critically reviewed and gave final approval of the manuscript; A. Prinz conceptualised the study and critically reviewed and gave final approval of the manuscript; G. Kahraman conceptualised the study and critically reviewed and gave final approval of the manuscript; $\mathrm{S}$. Barisic conceptualised the study and critically reviewed and gave final approval of the manuscript; O. Muftuoglu conceptualised the study and critically reviewed and gave final approval of the manuscript; N. Hirnschall conceptualised the study, performed statistical analysis, and critically reviewed and gave final approval of the manuscript.

\section{Data Availability Statement}

All data generated or analyzed during this study are included within the article. Further enquiries can be directed to the corresponding author.

\section{Funding Sources}

This research received no funding of any research relevant to the study.

\section{References}

1 Kinnersley P, Phillips K, Savage K, Kelly MJ, Farrell E, Morgan B, et al. Interventions to promote informed consent for patients undergoing surgical and other invasive healthcare procedures. Cochrane Database Syst Rev. 2013;7:CD009445.

2 Clark S, Mangram A, Ernest D, Lebron R, Peralta $\mathrm{L}$. The informed consent: a study of the efficacy of informed consents and the associated role of language barriers. J Surg Educ. 2011;68(2):143-7.

3 Patel DN, Wakeam E, Genoff M, Mujawar I, Ashley SW, Diamond LC. Preoperative consent for patients with limited English proficiency. J Surg Res. 2016;200(2):514-22.

4 Cassileth BR, Zupkis RV, Sutton-Smith K, March V. Informed consent: why are its goals imperfectly realized? N Engl J Med. 1980; 302(16):896-900.

5 Fink AS, Prochazka AV, Henderson WG, Bartenfeld D, Nyirenda C, Webb A, et al. Predictors of comprehension during surgical informed consent. J Am Coll Surg. 2010;210(6): 919-26.

6 Leclercq WK, Keulers BJ, Scheltinga MR, Spauwen PH, van der Wilt GJ. A review of surgical informed consent: past, present, and future. A quest to help patients make better decisions. World J Surg. 2010;34(7):1406-15.
7 Lee JS, Pérez-Stable EJ, Gregorich SE, Crawford MH, Green A, Livaudais-Toman J, et al. Increased access to professional interpreters in the hospital improves informed consent for patients with limited english proficiency. J Gen Intern Med. 2017;32(8):863-70.

8 Wollinger C, Hirnschall N, Findl O. Computer-based tutorial to enhance the quality and efficiency of the informed-consent process for cataract surgery. J Cataract Refract Surg. 2012;38(4):655-9.

9 Ghulam AT, Kessler M, Bachmann LM, Haller U, Kessler TM. Patients' satisfaction with the preoperative informed consent procedure: a multicenter questionnaire survey in switzerland. Mayo Clin Proc. 2006;81(3): 307-12.

10 Bundesministerium für europäische und internationale Angelegenheiten [Internet]. Migration \& Integration. Zahlen. Daten. Indikatoren; 2019 [cited 2020 Apr 29]. Available from: https://www.bmeia.gv.at/fileadmin/ user_upload/Zentrale/Integration/Integrationsbericht_2019/Migration-Integration-2019.pdf.

11 Karan A, Somasundaram P, Michael H, Shayegani A, Mayer H. The effect of multimedia interventions on the informed consent process for cataract surgery in rural South India. Indian J Ophthalmol. 2014;62(2):171-5.
12 Shukla AN, Daly MK, Legutko P. Informed consent for cataract surgery: patient understanding of verbal, written, and videotaped information. J Cataract Refract Surg. 2012; 38(1):80-4

13 Tipotsch-Maca SM, Varsits RM, Ginzel C, Vecsei-Marlovits PV. Effect of a multimediaassisted informed consent procedure on the information gain, satisfaction, and anxiety of cataract surgery patients. J Cataract Refract Surg. 2016;42(1):110-6.

14 Cornoiu A, Beischer AD, Donnan L, Graves $\mathrm{S}$, de Steiger R. Multimedia patient education to assist the informed consent process for knee arthroscopy. ANZ J Surg. 2011;81(3): $176-80$.

15 Ellett L, Villegas R, Beischer A, Ong N, Maher P. Use of a multimedia module to aid the informed consent process in patients undergoing gynecological laparoscopy for pelvic pain: randomized controlled trial. J Minim Invasive Gynecol. 2014;21(4):602-11.

16 Bollschweiler E, Apitzsch J, Apitsch J, Obliers R, Koerfer A, Mönig SP, et al. Improving informed consent of surgical patients using a multimedia-based program? Results of a prospective randomized multicenter study of patients before cholecystectomy. Ann Surg. 2008;248(2):205-11. 